Cahiers de la recherche sur les droits fondamentaux

$8 \mid 2010$

La liberté d'expression

\title{
La liberté d'expression : regards croisés sur ses sources, son contenu et ses fonctions
}

Michel Hottelier et Eleanor McGregor

\section{OpenEdition}

Édition électronique

URL : https://journals.openedition.org/crdf/6122

DOI : $10.4000 /$ crdf.6122

ISSN : 2264-1246

Éditeur

Presses universitaires de Caen

Édition imprimée

Date de publication : 20 décembre 2010

Pagination : 11-26

ISBN : 978-2-84133-367-7

ISSN : $1634-8842$

Référence électronique

Michel Hottelier et Eleanor McGregor, «La liberté d'expression : regards croisés sur ses sources, son contenu et ses fonctions ", Cahiers de la recherche sur les droits fondamentaux [En ligne], 8 | 2010, mis en ligne le 08 octobre 2020, consulté le 14 novembre 2022. URL : http://journals.openedition.org/crdf/ 6122 ; DOI : https://doi.org/10.4000/crdf.6122

Tous droits réservés 


\title{
La liberté d'expression : regards croisés sur ses sources, son contenu et ses fonctions
}

\author{
Michel HOTTELIER \\ Professeur à la Faculté de droit de l'Université de Genève \\ Eleanor MCGREGOR \\ Assistante à la Faculté de droit de l'Université de Genève
}

\author{
I. Les sources \\ II. La titularité \\ III. Le contenu \\ IV. Les effets \\ V. Les fonctions \\ VI. Les conditions d'exercice \\ VII. Les restrictions
}

\begin{abstract}
La libre communication des pensées et des opinions est un des droits les plus précieux de l'homme; tout citoyen peut donc parler, écrire, imprimer librement, sauf à répondre de l'abus de cette liberté dans les cas déterminés par la Loi.

Toute personne a droit à la liberté d'expression. Ce droit comprend la liberté d'opinion et la liberté de recevoir ou de communiquer des informations ou des idées sans qu'il puisse y avoir ingérence d'autorités publiques et sans considération de frontières.
\end{abstract}

1. Le premier de ces textes, chacun l'aura reconnu, est l'article 11 de la Déclaration des Droits de l'homme et du citoyen, du 26 août 1789 . Le second est l'article 11 de la Charte des droits fondamentaux de l'Union européenne, du 14 décembre 2007. Deux textes, deux numérotations d'articles identiques, mais deux époques bien distinctes.
L'une transpire la révolution, assimile l'homme au citoyen, parle d'un bien précieux et se méfie des abus. L'autre, d'essence internationale, énonce les principaux éléments de la liberté d'expression et transcende les frontières.

2. Les parallèles entre ces deux clauses fournissent l'opportunité de revenir aux éléments basiques de la liberté d'expression. Ils suscitent, partant, l'occasion de regards croisés. Pour ce faire, la présente contribution se propose de mettre en évidence ou de rappeler, à travers des illustrations empruntées tant au droit constitutionnel comparé qu'au droit international des Droits de l'homme, les traits saillants de cette garantie qui, pour avoir traversé les âges, demeure l'une des plus emblématiques de la protection de la personne humaine. Car même s'il est vrai que la convergence des systèmes juridiques libéraux en vue de proclamer l'importance essentielle de la liberté 
d'expression est totale, comme le souligne avec brio le professeur Patrick Wachsmann ${ }^{1}$, il n'est pas inutile de souligner les caractéristiques de la liberté d'expression telle qu'elle est reconnue, aménagée et mise en œuvre de nos jours.

\section{Les sources}

3. Les sources formelles de la liberté d'expression sont aujourd'hui multiples. Considérés, sous l'angle des sources matérielles, d'un point de vue historique, l'apparition et le développement de cette garantie trahissent un phénomène plus général, propre à la plupart des droits fondamentaux, que l'on peut résumer en quatre étapes : la naissance dans les doctrines politiques, la reconnaissance par le droit positif, la prise en main par le juge constitutionnel suivie de l'internationalisation de leur protection $^{2}$.

4. La naissance de la liberté d'expression dans les doctrines politiques s'inscrit dans le processus plus vaste de reconnaissance du rôle de l'individu au sein de la société civile et politique et, en particulier, son affranchissement du pouvoir, notamment de la puissance spirituelle. L'homme se voit ainsi progressivement impartir un rôle central dans l'organisation démocratique de la vie sociale. Acquis du courant des Lumières développé au cours du XVIII ${ }^{\mathrm{e}}$ siècle en Europe comme outre-Atlantique, la reconnaissance des libertés par le droit positif passe par l'affirmation, formelle et solennelle, d'un certain nombre de garanties individuelles, à l'image de la Déclaration française de $1789^{3}$. Il en va de même sur la scène internationale, où la garantie normative des Droits de l'homme a d'abord connu une phase purement proclamatoire, à l'image de la Déclaration universelle des Droits de l'homme du 10 décembre 1948. Ce processus se développe ensuite par la consécration, juridiquement contraignante cette fois, de la liberté d'expression dans des catalogues de droits individuels dotés de force constitutionnelle ou conventionnelle.

En précisant que le Congrès ne pourra faire aucune loi restreignant la liberté de parole ou de la presse, le premier amendement à la Constitution des États-Unis d'Amérique, adopté le 15 décembre 1791, est l'un des premiers instruments modernes imposant le respect de la liberté d'expression sur le terrain constitutionnel.

5. La reconnaissance des droits fondamentaux par des instruments normatifs appelés constitutions ne marque nullement le point d'aboutissement de ces garanties, mais bien plus leur point de départ sur le plan juridique.
C'est dire que leur prise en main par le juge constitutionnel représente l'étape suivante, destinée à leur conférer leur effet utile. Le contrôle de constitutionnalité, exercé par un corps institutionnel distinct du pouvoir politique, prolonge et complète la protection de ces garanties, contribuant ainsi à assurer la mise en œuvre de l'État fondé sur le droit. De fait, l'intervention de la justice constitutionnelle représente une nécessité consubstantielle à l'existence des libertés comme, plus largement, des droits fondamentaux dans leur ensemble.

Aux États-Unis, la garantie du free speech ne lie, à teneur du texte clair du premier amendement à la Constitution de 1787, que les autorités de l'Union, c'est-à-dire le Congrès et les autres "branches du Gouvernement", comme on dit là-bas. L'extension de sa protection aux États américains et, corrélativement, le contrôle de son respect par la Cour suprême ont été l'œuvre de cette dernière. Dans l'arrêt Gitlow qu'elle a rendu en $1925^{4}$, et qui portait sur la condamnation pénale d'un activiste sur la base d'une loi new-yorkaise réprimant l'anarchisme criminel, la Cour suprême a, à l'issue d'un intense débat doctrinal et jurisprudentiel ${ }^{5}$, imposé le respect du premier amendement aux États de l'Union en l'incorporant à la garantie de l'égale protection des lois (due process clause) opposable aux États au sens du $14^{\mathrm{e}}$ amendement adopté en 1868, à l'issue de la guerre de Sécession.

6. Il arrive que la liberté d'expression, à l'image d'autres droits fondamentaux, ne procède pas d'une reconnaissance impliquant des arbitrages de nature politique et l'adoption de normes de rang constitutionnel. Le juge peut, par une démarche finalement assez voisine du travail d'un constituant - ce qui ne va pas sans poser de délicats problèmes de légitimité -, reconnaître de lui-même des droits non écrits. Contrairement à une opinion communément répandue, ce processus de judge made law, c'est-à-dire de droit révélé par le juge, n'est pas propre aux États appartenant au système de la Common law.

En Suisse, par exemple, la liberté d'expression a été reconnue en 1961 par le Tribunal fédéral en tant que liberté non écrite relevant du droit constitutionnel fédé$\mathrm{ral}^{6}$. Cette reconnaissance ouvrait la porte à un contrôle de constitutionnalité sur le plan national, venant compléter la garantie des droits fondamentaux existant déjà au niveau des constitutions cantonales. En 1970, les juges fédéraux ont annulé pour la première fois une mesure cantonale au motif qu'elle contrevenait à la liberté d'expression, droit constitutionnel fédéral non écrit dont la liberté de la presse - alors garantie par l'article 55 de la Constitution fédérale (ci-après Cst.) - «est une des manifestations » ${ }^{7}$. Et en 1978, le Tribunal fédéral a rattaché

\footnotetext{
1. P. Wachsmann, «Liberté d'expression », in Dictionnaire des Droits de l’homme, J. Andriantsimbazovina et al. (éd.), Paris, PUF, 2008, p. 628.

2. Sur l'ensemble, voir A. Auer, G. Malinverni et M. Hottelier, Droit constitutionnel suisse, vol. II, Les Droits fondamentaux, $2^{\mathrm{e}}$ édition, Berne, Stämpfli, 2006 , p. 17.

3. M. Verpeaux, «Liberté d'expression et discours politique», AIJC, XXIII, 2007, p. 235.

4. 268 U.S. 652 Gitlow v. New York.

5. Voir, par exemple, 44 U.S. (3 How.) 589 Permoli v. New Orleans, où la Cour suprême déclare, en 1845, que la liberté religieuse consacrée par le premier amendement ne lie aucunement les États de l'Union. Voir également l'arrêt Barron v. Baltimore, 32 U.S. (7 Pet.) 243, rendu en 1833, qui consacre une vision strictement duale - opposant garanties de rang fédéral et de rang étatique - de la protection des droits constitutionnels.

6. Recueil officiel des arrêts du Tribunal fédéral suisse (ci-après ATF) 87 I 114 Sphinx Film S.A.

7. ATF 96 I 586 Aleinick.
} 
la liberté d'information aux libertés d'expression et de la presse $^{8}$.

Les conditions qui entourent la reconnaissance de droits fondamentaux par voie prétorienne sont strictes, de manière à éviter de court-circuiter le processus de révision formelle de la Constitution. Pour le Tribunal fédéral, il doit s'agir d'une faculté qui se présente comme une condition d'exercice d'autres libertés protégées par la Constitution ou qui constitue un élément essentiel de l'ordre démocratique fondé sur le droit. À ces prérequis s'ajoute l'exigence d'une certaine réalité constitutionnelle de la faculté en cause. Celle-ci doit donc se trouver déjà garantie par un certain nombre de constitutions cantonales ou résulter d'un large consensus en doctrine ${ }^{9}$.

7. En Suisse, depuis l'entrée en vigueur de la nouvelle Constitution fédérale, le $1^{\text {er }}$ janvier 2000 , la liberté d'expression fait partie d'une pluralité de garanties constitutionnelles qui ont toutes vocation à protéger la libre formation, la libre diffusion et la libre réception des opinions. La Constitution fédérale témoigne de cette diversité en regroupant, sous le couvert des libertés de communication, les libertés d'opinion et d'information (art. 16), la liberté des médias (presse, radio et télévision, ainsi que les « autres formes de diffusion de productions et d'informations ressortissant aux télécommunications publiques », art. 17), la liberté de la science (art. 20) et la liberté de l'art (art.21). S'ajoutent à cette panoplie d'autres garanties qui, en matière de diffusion des idées, jouent un rôle majeur, à l'image de la liberté de la langue (art. 18), des libertés de réunion (art. 22) et d'association (art. 23) ou encore du droit de pétition (art. 33) ${ }^{10}$.

La consécration distincte de plusieurs garanties dans le domaine de la communication - chacune étant dotée d'un champ d'application spécifique - procède d'un choix délibéré du constituant. Ce choix témoigne de l'évolution qui a caractérisé la liberté d'expression au cours des âges, en particulier sur le terrain doctrinal et jurisprudentiel, sans que l'on puisse inférer pour autant un ordre de priorité entre ces diverses garanties. De fait, la délimitation entre les domaines de protection de chacune des dispositions précitées n'est pas toujours aisée à établir, étant toutefois précisé que la liberté d'opinion et d'information énoncée à l'article 16 Cst. se présente sans doute comme la garantie à la fois générale et subsidiaire par rapport aux autres libertés de communication, lesquelles sont dotées de contours mieux définis eu égard aux comportements qu'elles appréhendent, comme le Tribunal fédéral l'a relevé ${ }^{11}$.

8. L'internationalisation de la protection des droits fondamentaux à travers la reconnaissance des Droits de l'homme représente une étape supplémentaire contribuant à une meilleure sauvegarde de la liberté d'expression. Depuis une soixantaine d'années, soit depuis la fin de la deuxième guerre mondiale, la dimension nationale de la protection des libertés les plus élémentaires s'est enrichie d'un étage de sauvegarde international, sur le plan aussi bien régional avec la Convention européenne des Droits de l'homme du 4 novembre 1950 qu'universel avec le Pacte international relatif aux droits civils et politiques du 16 décembre 1966. Sur le terrain juridique, deux questions au moins découlent de cette évolution.

9. La première concerne la nature des rapports entre la liberté d'expression telle qu'elle est traditionnellement protégée par le droit constitutionnel national et sa consécration, plus récente, à l'échelon international. S'agit-il de la même liberté, ou de formes distinctes, complémentaires voire, le cas échéant, antagoniques de droits individuels? Les droits constitutionnels de rang national convergent-ils entièrement avec leurs cousins qu'énoncent les instruments de protection des Droits de l'homme?

Ces questions peuvent appeler des réponses aussi bien affirmatives que négatives. À notre sens, il ne fait pas de doute que tant les droits constitutionnels d'origine nationale que les Droits de l'homme de rang international poursuivent l'objectif d'assurer une protection optimale, à leur niveau respectif, de l'individu dans ses rapports avec l'État. Certains éléments permettent toutefois de les distinguer. Là où les droits constitutionnels procèdent d'une vision et d'une culture essentiellement nationales, les Droits de l'homme découlent d'une prise de conscience, à l'échelon international, qui poursuit l'objectif d'assurer une certaine harmonisation et une protection minimale des garanties les plus élémentaires dont l'histoire de l'humanité a démontré, toujours de manière tragique, le haut degré de vulnérabilité. C'est dire que, loin de prétendre remplacer ou se substituer à la protection constitutionnelle, nationale par nature, des droits fondamentaux, la garantie internationale des Droits de l'homme tend à imposer aux États une protection minimale, donc subsidiaire, des droits individuels.

10. La seconde question que pose la coexistence d'un niveau national et d'un niveau international de protection des droits de la personne humaine concerne la nature du contrôle que requiert leur mise en œuvre. Là où, par nature, les droits individuels d'origine interne vont de pair avec une forme de juridiction constitutionnelle, la sauvegarde des Droits de l'homme protégés internationalement implique le développement d'un contrôle différent, complémentaire, le contrôle de la conventionnalité, ce dernier s'exerçant par ailleurs aussi bien à l'échelon national qu'international. Contrairement à une opinion communément répandue, la coexistence de ces deux types de contrôle n'est pas nécessairement toujours convergente.

En Suisse, l'article 190 Cst. fait obligation au Tribunal fédéral et aux autres autorités d'appliquer les lois

8. ATF 104 Ia 88 Bürgin. Plus généralement, voir A. Auer, G. Malinverni et M. Hottelier, Droit constitutionnel suisse, p. 35.

9. Voir, par exemple, l'arrêt Kaufmann ATF 100 Ia 392, à propos de la reconnaissance - refusée en la circonstance - de la liberté de manifestation sur le domaine public.

10. Sur l'ensemble, voir A. Auer, G. Malinverni et M. Hottelier, Droit constitutionnel suisse, p. 249.

11. ATF 127 I 145 Wottreng. 
votées par l'Assemblée fédérale. La disposition est traditionnellement interprétée comme interdisant aux juges de refuser d'appliquer une loi fédérale au motif que l'une ou l'autre de ses dispositions contreviennent à la Constitution. Le Tribunal fédéral est ainsi tenu d'appliquer une loi fédérale, que celle-ci soit conforme ou contraire à la liberté d'expression telle qu'elle est garantie par la Constitution. Mais l'article 190 Cst. fait également obligation au Tribunal fédéral d'appliquer le droit international, soit en particulier les instruments de protection des Droits de l'homme auxquels la Suisse est partie. Dans l'hypothèse où une loi fédérale contrevient à un droit de l'homme, le Tribunal fédéral admet de nos jours, au prix d'une jurisprudence qui a considérablement évolué depuis la ratification de la Convention européenne des Droits de l'homme (ci-après CEDH) par la Suisse, que c'est la garantie conventionnelle qui prime. En d'autres termes, là où le contrôle de la constitutionnalité n'est pas possible, le contrôle de la conventionnalité est, lui, pratiqué. Contrairement à d'autres États, par exemple à la Belgique $^{12}$, les instruments internationaux de protection des Droits de l'homme et les droits fondamentaux d'origine nationale ne forment donc pas, en Suisse, un ensemble indissociable conduisant au même type de contrôle. Cette situation singulière permet du même coup des discordances entre le contrôle de constitutionnalité et celui de la conventionnalité.

11. Grâce au contrôle de la conventionnalité, la liberté d'expression rencontre ainsi un soutien de poids, alors même que le contrôle de la constitutionnalité est toujours verrouillé en droit suisse par la clause de l'article 190 Cst. $^{13}$.

Le Tribunal fédéral a, par exemple, jugé que la répression pénale de la diffusion de magazines et de cassettes vidéo, qui ont comme contenu des actes d'ordre sexuel avec des excréments humains ou qui comprennent des actes de violence, n'est pas contraire à la liberté d'expression au sens de l'article $10 \mathrm{CEDH}$, même si ces produits sont proposés exclusivement à des adultes initiés ${ }^{14}$.

12. Le regard porté par l'instance chargée de statuer sur une éventuelle violation de la liberté d'expression peut être fort différent selon que celui-ci se situe dans le contexte propre au droit constitutionnel, donc au droit interne, ou au contraire sur le plan international. Les règles de procédure applicables à chacun de ces niveaux peuvent exercer une influence déterminante sur leur résolution. De fait, le contrôle interne de la constitutionnalité et le contrôle de la conventionnalité peuvent entrer en conflit. La nature du contrôle exercé n'est ainsi pas dépourvue d'incidence sur le choix de la norme de référence jugée prioritaire.

Dans l'affaire Hertel c. Suisse par exemple, un scientifique qui avait réalisé une enquête sur les effets potentiellement cancérigènes des aliments préparés au moyen de fours à micro-ondes se plaignait d'une restriction à sa liberté d'expression en raison de l'interdiction signifiée à un journal de publier des extraits de son étude, aux fins de protéger la liberté économique des fabricants d'appareils de ce genre ${ }^{15}$. L'affaire opposait, pour la circonstance, la liberté d'expression au sens de l'article 10 CEDH à la liberté économique au sens de l'article 27 Cst.

Saisi du litige, le Tribunal fédéral fit sans hésitation primer la seconde sur la première, privilégiant ainsi la garantie constitutionnelle d'origine interne sur la norme conventionnelle. Le respect de la Constitution suisse et de la législation fédérale sur la concurrence déloyale - en particulier, le respect d'une loi fédérale récemment adoptée par les Chambres fédérales - l'emporta sans difficulté devant la Cour suprême helvétique sur le respect du droit européen. Le maigre considérant de l'arrêt consacré à cet aspect du dossier trahit sans ambiguïté les préférences affichées par la Haute Cour à la primauté du droit national, soit à la prééminence de la loi fédérale au sens de l'article 190 Cst. destinée, en l'espèce, à lutter contre la concurrence déloyale.

Saisie à son tour, la Cour européenne des Droits de l'homme formula une opinion diamétralement opposée sous l'angle de l'article 1o CEDH. Constatant une violation de cette garantie en raison de l'impossibilité faite au requérant de voir son étude publiée, fût-ce par voie d'extraits, la Cour condamna la Suisse. Le contrôle de la conventionnalité pratiqué par les juges européens au sens des articles 1 et $19 \mathrm{CEDH}$, allié à l'absence de garantie de la liberté économique dans la Convention, prima ainsi sur le contrôle effectué par les juges helvétiques. La différence affectant la nature du contrôle exercé par le juge suisse sur la base de l'article 190 Cst., d'une part, et par le juge européen, d'autre part, permet d'expliquer la différence d'approche et, finalement, de solution.

\section{La titularité}

13. La liberté d'expression est une liberté individuelle par nature. Elle se distingue à cet égard d'autres catégories de Droits de l'homme comme les garanties de l'État de droit, qui ne tendent pas à la protection de comportements humains déterminés, mais prescrivent à l'État la manière dont il doit s'organiser et accomplir ses fonctions ${ }^{16}$. Quelques-uns des cas d'application les plus célèbres de la liberté d'expression ont permis de cerner plus précisément ses contours et le cercle de ses bénéficiaires.

Dans un arrêt rendu en 1969, en pleine vague de contestation de la guerre du Vietnam, la Cour suprême américaine a eu l'occasion de préciser que la garantie du free speech trouvait matière à s'appliquer dans le contexte

12. Voir F. Delpérée, «Constitution et liberté d'expression. Rapport belge », AIJC, XXIII, 2007, p. 133.

13. Sur la question, voir M. Hottelier, «Le système suisse de justice constitutionnelle», in Dignidad de la persona, Derechos fundamentales, Justicia constitucional, F. Fernandez Segado (éd.), Madrid, Dykinson-Constitucional, 2008, p. 852.

14. ATF 128 IV 201 X., Y. und Z.

15. Cour EDH, Hertel c. Suisse, 25 août 1998, Rec., 1998-VI.

16. Sur les différentes catégories de Droits de l'homme, voir A. Auer, G. Malinverni et M. Hottelier, Droit constitutionnel suisse, p. 8. 
scolaire aussi bien à l'égard des élèves que des enseignants. Pour les juges de Washington, le portail des établissements d'enseignement ne représente ainsi nullement un rempart contre l'exercice de cette liberté. En l'espèce, la Cour suprême a annulé la sanction disciplinaire prise à l'encontre d'un collégien qui, en signe de protestation contre l'engagement des forces armées américaines au Vietnam, avait arboré un brassard noir à l'école ${ }^{17}$.

14. De même, outre les écoliers, les lycéens ou les étudiants, les personnes employées par l'État (membres de la fonction publique) ou celles qui lui sont liées par un rapport juridique d'une intensité particulière, comme les personnes privées de liberté ou les soldats, restent titulaires de la liberté d'expression. L'accomplissement des tâches étatiques peut toutefois requérir, dans ce cadre, l'adoption de mesures restrictives d'une nature spécifique.

La Cour suprême américaine a jugé que, tout comme le portail de l'école, les murs d'une prison ne font pas obstacle à l'invocation de la liberté d'expression ${ }^{18}$.

15. La question peut se poser, en sens inverse, de savoir si certaines personnes disposent d'un statut privilégié dans l'exercice de la liberté d'expression, à l'instar des journalistes. La réponse n'est pas univoque, mais varie en fonction des traditions juridiques.

De jurisprudence constante, la Cour européenne des Droits de l'homme accorde à la presse, «à qui il appartient de communiquer des informations et des idées sur les questions débattues dans l'arène politique, tout comme sur celles qui concernent d'autres secteurs d'intérêt public» une importance particulière lorsqu'il s'agit de diffuser des informations «qui heurtent, choquent ou inquiètent ${ }^{19}$. La notion de presse ne se limite toutefois pas aux seuls journalistes professionnels, mais s'étend à un spectre plus large de particuliers désireux de faire connaître leur point de vue. C'est ainsi que les militants non strictement politiques ont pu bénéficier d'un traitement similaire à celui de la presse ${ }^{20}$.

La question est controversée en doctrine américaine. Si l'importance historique de la liberté de la presse est indéniable - le Bill of Rights ayant été envisagé pour protéger la diffusion d'information par cette voie -, il n'est pas certain que les médias bénéficient d'un statut particulier. Repérer un traitement différencié n'est toutefois guère aisé en pratique étant donné le champ d'application très étendu accordé à la liberté d'expression, notamment en matière de diffamation.
16. Les personnes morales sont bien entendu, elles aussi, titulaires de la liberté d'expression.

Dans l'affaire VgT Verein gegen Tierfabriken c. Suisse, une association s'est prévalue devant la Cour européenne des Droits de l'homme, puis devant la Grande Chambre, d'une violation de l'article 10 $\mathrm{CEDH}$ en raison du refus qui lui avait été signifié de diffuser un spot télévisé consacré aux conditions d'élevage industriel des porcs $^{21}$. La Cour a même eu l'occasion de préciser, dans une affaire opposant une organisation non gouvernementale à la République de Lettonie, que la participation d'une association au débat public est «essentielle pour une société démocratique $»^{22}$.

\section{Le contenu}

17. Le champ opératoire de la liberté d'expression est étendu. Les pensées, les idées, les opinions ou les informations: chacun de ces éléments, qui se décline à son tour en une multitude de variantes, bénéficie de sa protection. La protection du débat politique, élément fondateur des États démocratiques, occupe une place centrale dans ce cadre.

La Cour européenne des Droits de l'homme considère que l'article 10 paragraphe $2 \mathrm{CEDH}$ ne laisse guère de place pour des restrictions à la liberté d'expression dans le domaine du discours politique ou de questions d'intérêt général. Elle fait preuve de la plus grande prudence lorsque les mesures prises ou les sanctions infligées par les autorités nationales sont de nature à dissuader les médias de participer à la discussion de problèmes d'un intérêt général légitime ${ }^{23}$.

L'article $10 \mathrm{CEDH}$ protège aussi les messages commerciaux $^{24}$. La Cour suprême des États-Unis ne dit pas autre chose ${ }^{25}$, tout en considérant qu'en raison de la faiblesse de leur apport au discours sociétal, les messages publicitaires prêtent relativement facilement le flanc à restriction ${ }^{26}$.

18. Comme l'a jugé le Conseil constitutionnel français, tous les aspects propres à la liberté de communication bénéficient de la protection constitutionnelle qu'offre l'article 11 de la Déclaration des Droits de l'homme et du citoyen, même s'ils empruntent la forme de supports techniques encore inconnus en $1789^{27}$.

La portée très générale conférée à cette garantie rend ainsi inutile, à l'intérieur de son champ d'application

\footnotetext{
17. 393 U.S. 503 (1969) Tinker v. Des Moines School Dist.

18. 482 U.S. 78 (1987) Turner v. Safley.

19. Cour EDH, Lopes Gomes da Silva c. Portugal, 28 septembre 2000, Rec., 2000-X, \$30.

20. Cour EDH, Steel et Morris c. Royaume-Uni, 15 février 2005, Rec., 2005-II. Voir aussi P.-F. Docquir, Variables et variations de la liberté d'expression aux États-Unis, Bruxelles, Bruylant - Nemesis (Droit et justice, $\mathrm{n}^{\circ} 72$ ), 2007, p. 81.

21. Cour EDH, Verein gegen Tierfabriken Schweiz c. Suisse, 30 juin 2009.

22. Cour EDH, Vides Aizsarbzibas Klubs c. Lettonie, 27 mai 2004, $\$ 42$.

23. Voir, entre autres, Cour EDH, Monnat c. Suisse, 21 septembre 2006, $\$ 58$.

24. Cour EDH, Markt intern Verlag c. Allemagne, 20 novembre 1989, série A, nº 165; Casado Coca c. Espagne, 24 février 1994, série A, $\mathrm{n}^{\circ} 285-\mathrm{A}$.

25. 319 U.S. 105 (1943) Murdock v. Pennsylvania.

26. 492 U.S. 469 (1989) Board of Trustees of State University of New York.

27. Décision du 27 juillet 1982 relative à la loi sur la communication audiovisuelle.
} 
matériel, l'appel aux principes fondamentaux reconnus par les lois de la République ${ }^{28}$.

19. La liberté d'expression recouvre également la diffusion de messages, d'idées ou de points de vue selon d'autres modes que l'expression orale ou écrite.

Outre le port d'un brassard destiné à marquer la désapprobation qu'entend exprimer son porteur à l'égard d'un conflit, la Cour suprême américaine a par exemple admis que le port d'une veste sur laquelle était inscrite la phrase "fuck the draft» pour s'opposer à l'enrôlement dans les forces armées bénéficiait de la protection du premier amendement ${ }^{29}$. De même, celui qui brûle ses papiers militaires en signe de protestation et pour faire étal de ses convictions pacifistes peut se prévaloir de la liberté d'expression ${ }^{30}$, tout comme celui qui met le feu à la bannière du pays ${ }^{31}$. Ou encore, le fait de dormir sur les pelouses qui jouxtent la Maison Blanche constitue une activité couverte par le premier amendement $^{32}$.

En Suisse également, la notion d'expression se décline de manière extensive dans le domaine politique comme dans les autres domaines. Le Tribunal fédéral a, au cours d'une jurisprudence abondante, eu l'occasion d'en préciser les contours, en relevant entre autres que les banderoles ${ }^{33}$, les drapeaux ${ }^{34}$ ou encore les masques arborés lors de manifestations sur le domaine public ${ }^{35}$, pour véhiculer des messages déterminés, font tous partie intégrante des libertés de communication. Même le fait de bloquer temporairement le trafic afin de protester contre la guerre a été considéré comme une forme d'expression, tout comme la confection d'un tapis humain destiné à entraver l'accès à une exposition d'armes ${ }^{36}$.

20. Ainsi comprise, la liberté d'expression ne protège pas seulement le contenu des informations, mais également leurs moyens et leurs modalités de transmission et de réception.

Une interdiction généralisée d'utiliser des hautparleurs à l'occasion de rassemblements politiques a, par exemple, été considérée comme inconstitutionnelle par le Tribunal fédéral suisse ${ }^{37}$. En revanche, les actes de vandalisme comme l'utilisation de sprays, la casse de vitrines, le harcèlement des passants ou la destruction de véhicules ne font pas partie de la liberté d'expression ${ }^{38}$.

21. Toutes les opinions sont-elles pour autant appréhendées par la liberté d'expression? Ce thème récurrent alimente une pratique soutenue et variée. Deux théories s'affrontent sur le sujet. La première considère que le champ de protection de la liberté d'expression rencontre inévitablement des limites, en sorte que certains messages ne sauraient faire partie de ses éléments constitutifs. La seconde procède d'une vision plus large du champ opératoire de cette liberté. Le problème se situe alors non pas sous l'angle de l'objet ou du contenu des messages protégés, mais sous l'angle des restrictions susceptibles d'être opposées à cette garantie.

Dans un arrêt du 12 juillet 1996, la Cour constitutionnelle belge a jugé que la répression pénale des propos révisionnistes ne contrevenait pas à la liberté d'expression. L'intervention répressive du législateur est en effet justifiée lorsqu'un droit fondamental est exercé d'une manière telle que les principes de base de la société démocratique s'en trouvent menacés et qu'il en résulte un dommage inacceptable pour autrui. La Cour a ajouté que le législateur belge pouvait légitimement redouter que la Belgique devienne un refuge du négationnisme en l'absence de législation de ce genre ${ }^{39}$.

De son côté, la Cour suprême canadienne estime que la liberté d'expression n'est pas violée par le rejet de la propagande haineuse. À son sens, il s'agit, au contraire, du «meilleur moyen dont dispose l'État pour encourager la protection de valeurs qui sont l'essence même de la liberté d'expression, tout en manifestant son aversion pour la vision préconisée par les fondements de haine ${ }^{40}$.

En Suisse, le Tribunal fédéral a jugé que la norme pénale réprimant la haine et la discrimination raciale, ethnique ou religieuse rentrait dans le cadre des restrictions à la liberté d'expression figurant à l'article 10 paragraphe 2 CEDH. La Haute Cour a en conséquence confirmé la condamnation d'une personne pour avoir publié des articles de propagande raciste et d'atteinte à la mémoire du génocide de la seconde guerre mondiale ${ }^{41}$.

La Cour européenne des Droits de l'homme cultive une autre approche du champ d'application de la liberté d'expression. Dans la décision d'irrecevabilité qu'elle a rendue le 24 juin 2003 dans l'affaire Garaudy c. France, elle a jugé que les propos négationnistes ne font pas partie de la liberté protégée par l'article 10 CEDH. En dépit du caractère éminent et essentiel que revêt la liberté d'expression dans une société démocratique, il ne fait

\footnotetext{
28. P. Wachsmann, Libertés publiques, $6{ }^{\mathrm{e}}$ édition, Paris, Dalloz, 2009, p. 569.

29. 403 U.S. 15 (1971) Cohen v. California.

30. 391 U.S. 367 (1968) United States v. O’Brien.

31. 496 U.S. 310 (1990) United States v. Eichmann; 491 U.S. 397 (1989) Texas v. Johnson.

32. 468 U.S. 288 (1984) Clark v. Community for creative non-violence.

33. ATF 111 Ia $322 I$.

34. ATF 107 Ia 59 L'Amicale des patoisants de la Prévôté.

35. ATF 117 Ia 472 Sozialdemokratische Partei Basel-Stadt.

36. ATF 119 IV $301 X$. ; voir également ATF 108 IV 165 M.

37. ATF 107 Ia 64 Progressive Organisationen Basel.

38. ATF 111 Ia $322 I$.

39. Arrêt n ${ }^{\circ}$ 45/96, cité et commenté par F. Moline dans Les Grandes Décisions des cours constitutionnelles européennes, P. Bon et D. Maus (éd.), Paris, Dalloz, 2008, p. 289.

40. R. c. Keegstra, 1990/3, Recueil des arrêts de la Cour suprême du Canada (ci-après RCS) 697, p. 77.

41. Arrêt 6P.92/2001 Gaston-Armand Amaudruz, 16 octobre 2001
} 
aucun doute qu'à l'égal de tout autre propos dirigé contre les valeurs qui sous-tendent la Convention, la justification d'une politique pronazie ne saurait bénéficier de la protection qu'octroie l'article $10 \mathrm{CEDH}$. Il existe, pour les juges de Strasbourg, une catégorie de faits historiques clairement établis - comme l'Holocauste -, dont la négation ou la révision est soustraite par l'article $17 \mathrm{CEDH}$ à la protection de l'article 10 CEDH. La liberté d'expression ne saurait par conséquent trouver matière à s'appliquer dans ce cadre ${ }^{42}$. De fait, à l'égal de tout autre propos dirigé contre les valeurs qui sous-tendent la Convention, la justification d'une politique pronazie ne saurait bénéficier de la protection de l'article $10 \mathrm{CEDH}^{43}$.

22. Aux États-Unis, l'étendue de la protection conférée aux individus dépend de la catégorie dans laquelle tombe l'expression: si l'incitation à la violence, la diffamation, l'obscénité, et les injures échappent à toute protection, les autres expressions en bénéficient pleinement.

Le discours raciste ne figure pas parmi les expressions jugées inconstitutionnelles, de sorte qu'il emporte une protection très large. Par ailleurs, l'inconstitutionnalité, en droit américain, du discours fondé sur le contenu empêche la criminalisation de la propagande haineuse. Les tentatives dans ce sens ont, pour la plupart, échoué ${ }^{44}$. Deux arrêts ont toutefois ouvert une brèche, en laissant entendre qu'une restriction imposée à la liberté d'expression se justifie dans le cas d'un discours de haine impliquant un risque de violence ${ }^{45}$.

23. La question des publications heurtant la morale alimente également une casuistique variée sous l'angle du contenu et des limites opposables à la liberté d'expression.

Un arrêt rendu le 3 mai 2007 a permis au Tribunal fédéral suisse de prendre position sur la diffusion de messages publicitaires vantant le téléchargement de vidéos et d'images pornographiques destinées aux téléphones portables. Après s'être interrogé sur les notions de moralité publique et de pornographie au sens du droit des médias, la Haute Cour a précisé que, dans la mesure où ils donnent, par l'image et le son, une représentation vulgaire et primitive de la personne humaine qui réduit celle-ci au simple rôle d'objet sexuel ayant perdu toute parcelle d'humanité, les spots publicitaires litigieux - et pas seulement les produits que ceux-ci tendaient à promouvoir - allaient au-delà de ce qui est juridiquement admissible en matière de contenu érotique sous l'angle de la protection de la jeunesse. L'interdiction faite à une télévision privée de diffuser ce genre de messages a ainsi été confirmée sous l'angle de la liberté d'expression ${ }^{46}$.

Dans le même sens, la Chambre des lords a estimé que l'interdiction de sex-shops dans les quartiers d'habitation était compatible avec la liberté d'expression. Selon Lord Hoffmann, il est douteux que la distribution de livres pornographiques soit couverte par la liberté d'expression. Si l'article $10 \mathrm{CEDH}$ trouve, malgré tout, application, ce ne peut être « qu'à un degré très minime » ${ }^{47}$.

La question avait déjà été soulevée en 1988 devant la Cour européenne des Droits de l'homme, sous l'angle de la liberté de l'art. Cette affaire concernait la confiscation, par les autorités locales, de toiles exposées par un artiste peintre, représentant "crûment des relations sexuelles, en particulier entre hommes et animaux ", ainsi que sa condamnation à une amende pour publications obscènes. Les juges de Strasbourg se sont prononcés en faveur des autorités nationales, estimant que de telles mesures étaient nécessaires dans une société démocratique, eu égard, notamment, à la marge d'appréciation qui revient aux États contractants dans le domaine de la morale ${ }^{48}$.

24. Lorsque les opinions cèdent leur place aux injures ou à des propos offensants et diffamatoires, une difficulté supplémentaire surgit sous l'angle de la protection conférée par la liberté d'expression. De manière générale, l'attitude de la Cour européenne est plutôt libérale. La protection déploie également ses effets lorsque la critique est de nature à discréditer une personnalité politique.

De l'avis de la Cour, «l'homme politique s'expose inévitablement et consciemment à un contrôle attentif de ses faits et gestes, tant par les journalistes que par la masse des citoyens, et doit montrer une plus grande tolérance, surtout lorsqu'il se livre lui-même à des déclarations publiques pouvant prêter à critique. »

L'instance alsacienne poursuit en relevant que «dans ce domaine l'invective politique déborde souvent sur le plan personnel : ce sont là les aléas du jeu politique et du libre débat d'idées, garants d'une société démocratique ${ }^{49}$. Ainsi, qualifier un dirigeant politique d' «imbécile» ne justifie pas en soi une restriction à la liberté d'expression. Il en va de même des termes "grotesque», "rustre» et «grossier» apposés à un avocat et journaliste en raison de ses idées politiques ${ }^{50}$ et des expressions à connotation criminelle telles qu' «escroquer», «voler», «dérober», « arnaque employés à l'encontre d'un ministre des Finances alors qu'il n'avait jamais été poursuivi pénalement

42. Requête n ${ }^{\circ}$ 65831/o1 Roger Garaudy c. France, décision du 24 juin 2003, Rec., 2003-IX, p. 361.

43. Cour EDH, Lehideux et Isorni c. France, 23 septembre 1998, Rec., 1998-VII, $\$ 53$.

44. Pour plus de détails, voir J. Bell, « Pour faire barrage à ceux qui n’ont pas de cœur : expressions racistes et droit des minorités », in La Liberté d'expression aux États-Unis et en Europe, E. Zoller (éd.), Paris, Dalloz, 2008, p. 58 sq.

45. 508 U.S. 476 (1993) Wisconsin v. Mitchell; 538 U.S. 343 (2003) Virginia v. Black.

46. ATF 133 II 136 Star TV AG.

47. Belfast City Council v. Miss Behavin' Limited [2007] UKHL 19; pour plus de détails, voir E. Barendt, "La liberté d'expression au Royaume-Uni et le Human Rights Act de 1998 ", in La Liberté d'expression aux États-Unis et en Europe, p. 42.

48. Cour EDH, Müller et autres c. Suisse, 24 mai 1988, série A, $n^{\circ} 133$.

49. Cour EDH, Lopes Gomes da Silva c. Portugal, 28 septembre 2000, Rec., 2000-X, \$30; voir aussi Cour EDH, Cuc Pascu c. Royaume-Uni, 16 septembre $2008, \$ 34$.

50. Cour EDH, Lopes Gomes da Silva c. Portugal, 28 septembre 2000, Rec., 2000-X. 
pour les agissements en cause ${ }^{51}$, ou encore «Bonnie and Clyde » pour caractériser un membre du Parlement en voyage avec sa compagne au Brésil ${ }^{52}$.

Il existe toutefois, dans la jurisprudence de la Cour de Strasbourg, une soupape de sécurité en faveur des hommes politiques à travers l'exigence imposant de conserver un minimum de modération et de bienséance. C'est ainsi qu'assimiler un homme politique à un «chef de bande de tueurs», affirmer que l'assassinat perpétré par un personnage même de fiction a été " recommandé» par lui et le qualifier de "vampire qui se nourrit de l'aigreur de ses électeurs mais aussi parfois de leur sang", ne saurait être couvert par la liberté d'expression ${ }^{53}$.

25. Bien que dans une moindre mesure, les personnalités non politiques doivent, elles aussi, accepter une certaine dose de critique dans la mesure où leur statut ou les fonctions qu'elles exercent les exposent à un large public.

La Cour européenne des Droits de l'homme a eu l'occasion de relever cela à l'égard de policiers ${ }^{54}$, d'un professeur d'université ${ }^{55}$, et même d'un archevêque ${ }^{56}$.

26. La position de la Cour européenne rejoint celle de la Cour suprême américaine, laquelle fait preuve d'une tolérance éprouvée face au discours insultant, voire outrageant ${ }^{57}$. Aux États-Unis en effet, le domaine couvert par la liberté d'expression permet d'englober la quasi-totalité des expressions du cœur et de l'esprit, et ce par une variété infinie de moyens ${ }^{58}$. De fait, diffamer un personnage officiel n'échappe pas en soi à la protection de la liberté d'expression. Seuls les propos tenus de manière à troubler l'ordre public, en provoquant par hypothèse des émeutes, sont exclus de la protection qu'offre le premier amendement. Ces expressions tombent alors sous la catégorie très étroite des fighting words ${ }^{59}$. Hormis cette hypothèse, les particuliers sont libres d'exprimer leurs opinions comme ils l'entendent. Dans cette perspective, employer des termes insultants à l'endroit de policiers en fonction est protégé par la liberté d'expression ${ }^{60}$.

Au Canada, la Cour suprême a souligné que le droit de s'exprimer politiquement commande un haut degré de protection constitutionnelle. La limite se situe au niveau des manifestations violentes. L'entartage d'un politicien a ainsi été considéré comme ne bénéficiant d'aucune protection constitutionnelle ${ }^{61}$.

27. Qu'en est-il lorsqu'un particulier révèle des informations confidentielles? De manière générale, les instances juridictionnelles s'accordent à admettre que de telles informations sont couvertes par la liberté d'expression. Cela étant, les positions divergent sur le terrain des restrictions opposables à cette garantie.

En 2003, la Chambre des lords a été saisie de la question de la compatibilité des dispositions d'une loi de 1989 sur les secrets d'État avec la liberté d'expression ${ }^{62}$. Après avoir constaté que cette loi constituait une limitation de la liberté d'expression, la Chambre des lords a estimé que cette ingérence satisfaisait pleinement aux conditions de restriction. L'interdiction de divulguer des secrets officiels ne présentait toutefois pas un caractère absolu, la loi prévoyant une série de mesures à disposition du particulier en cas de doutes sur le fonctionnement du service ${ }^{63}$.

Dans le même sens, la Grande Chambre de la Cour européenne des Droits de l'homme a récemment renversé un arrêt de Chambre pour conclure à l'absence de violation de l'article 1o CEDH. Elle a estimé que l'amende infligée à un journaliste pour avoir publié des extraits d'un rapport confidentiel rédigé par l'ambassadeur de Suisse aux États-Unis au sujet de la stratégie à suivre dans le cadre de l'affaire des fonds bancaires de victimes de l'Holocauste durant les années 1990 était conforme au principe de proportionnalité ${ }^{64}$. Contrairement à la Chambre, la Grande Chambre a prêté une importance décisive à la forme de la publication qu'elle considérait comme «tronquée et réductrice» et "de nature à induire en erreur les lecteurs au sujet de la personnalité et des aptitudes de l'ambassadeur».

La Cour suprême américaine adopte, quant à elle, une position plus tranchée: "a stranger's illegal conduct does not suffice to remove the First Amendment shield from speech about a matter of public concern ${ }^{65}$."

28. Une partie non négligeable de la protection qu'octroie la liberté d'expression concerne le statut des

51. Cour EDH, A/S Diena et Ozolins c. Lettonie, 12 juillet 2007.

52. Cour EDH, Wirtschafts-Trend Zeitschriften-Verlagsgesellschaft M.B.H c. Autriche, 13 décembre 2005.

53. Cour EDH, Lindon, Otchakovsky-Laurens et July c. France, 22 octobre 2007. Pour un commentaire de cet arrêt, voir P. Wachsmann, « Vers un affaiblissement de la protection de la liberté d'expression par la Cour européenne des Droits de l'homme? ", Revue trimestrielle des Droits de l'homme, $\mathrm{n}^{\circ}$ 79, 2009, p. 491

54. Selon la Cour, ne constitue pas un langage excessif le fait de traiter des policiers suspectés de brutalités de "bêtes en uniforme», de «fauves en uniforme », de «brutes sadiques»; voir Cour EDH, Thorgeir Thorgeirson c. Islande, 25 juin 1992, série A, $\mathrm{n}^{\circ} 239$.

55. Cour EDH, Brunet-Lecomte et Sarl Lyon Mag' c. France, 20 novembre 2008.

56. Cour EDH, Klein c. Slovaquie, 31 octobre 2006.

57. 337 U.S. 1 (1949) Terminiello v. Chicago; 403 U.S. 15 (1971) Cohen v. California; 485 U.S. 46 (1988) Hustler Magazine v. Falwell; 485 U.S. 312 (1988) Boos v. Barry.

58. L. Pech, «Constitution et liberté d'expression. Rapport des États-Unis », AIJC, XXIII, 2007, p. 181.

59. M. Hottelier, «La liberté d'expression aux États-Unis : une visite guidée », PJA, 1999, p. 8.

60. 405 U.S. 518 (1972) Gooding v. Wilson; 415 U.S. 130 (1974) Lewis v. New Orleans.

61. R. v. Geoghegan (2005) C.C.C. (3d) 85 .

62. Chambre des lords, R. v. Shayler (2003) 1 AC 247.

63. E. Barendt, «La liberté d'expression au Royaume-Uni... », p. 34 sq.

64. Cour EDH, Stoll c. Suisse, 10 décembre 2007 ; M. Hottelier, « La liberté de la presse entre confidentialité et provocation : mode d'emploi pour faire chuter une liberté de son piédestal. Cour européenne des Droits de l'homme (Grande Chambre), Stoll c. Suisse, 10 décembre 2007 ", Revue trimestrielle des Droits de l'homme, $\mathrm{n}^{\circ} 75,2008$, p. 801.

65. 532 U.S. 514 (2001) Bartnicki v. Vopper. 
journalistes. Ces derniers peuvent-ils être tenus de dévoiler leurs sources, en particulier dans le cadre de procédures pénales? Les réponses que fournit la jurisprudence sur le sujet ne sont pas uniformes.

Alors qu'aux États-Unis, la conception traditionnelle a tendance, au nom du principe d'égalité de traitement, à ne pas privilégier la protection des sources journalistiques ${ }^{66}$, la Cour européenne des Droits de l'homme range le secret des sources des journalistes parmi les éléments constitutifs de la liberté d'expression pour en faire même l'une des pierres angulaires de la liberté de la presse. En Suisse, conformément à l'article 17 alinéa 3 Cst., le secret de rédaction est protégé. La Constitution fédérale a ainsi pris en compte les enseignements qui découlent de la jurisprudence développée par la Cour européenne des Droits de l'homme ${ }^{67}$. Pour bénéficier de la protection de la Constitution, cet aspect de la liberté d'expression n'est cependant pas absolu, des restrictions pouvant lui être opposées moyennant une pesée des intérêts en cause, conformément à l'article 36 Cst.

Dans un arrêt du 11 mai 2006, le Tribunal fédéral a précisé les contours du droit à la protection des sources journalistiques. À l'issue d'une analyse approfondie des intérêts en cause, l'intérêt consistant à élucider les circonstances d'un décès provoqué par une erreur médicale à l'hôpital universitaire de Zurich n'a pas été considéré comme prépondérant pour permettre de contraindre le journaliste en cause à révéler la source des informations dont il disposait sur le sujet. Les circonstances du litige étaient en effet suffisamment établies sans qu'il s'avère nécessaire de recourir à cette mesure dans le cadre de la procédure pénale ${ }^{68}$.

29. Enfin, la publicité à caractère politique est-elle couverte par la liberté d'expression ? À en croire la jurisprudence de la Cour européenne des Droits de l'homme, la réponse est positive.

Dans un arrêt qui remonte à 2001, la Cour a déclaré contraire à l'article $10 \mathrm{CEDH}$ le fait d'interdire un spot publicitaire ayant pour but de dénoncer l'élevage en batterie des porcs et d'exhorter le public à consommer moins de viande. Une telle interdiction empêchait le débat public et ne répondait pas à l'exigence de nécessité dans une société démocratique ${ }^{69}$.

Cet arrêt a été accueilli avec scepticisme au RoyaumeUni. Dans une affaire similaire, la Chambre des lords n'a pas suivi le raisonnement de la Cour européenne ${ }^{70}$. L'affaire portait en l'occurrence sur l'interdiction de diffuser une publicité à contenu politique sur la télévision britannique. Le spot publicitaire mettait en scène une petite fille enchaînée dans la pénombre d'une cage pour animaux. Il s'inscrivait dans le cadre d'une campagne intitulée My Mate's a Primate [Mon ami est un primate], qui avait pour objectif de dénoncer l'exploitation des primates par les humains, ainsi que le risque d'extinction auquel cette espèce est exposée. Contrairement à l'avis de la Cour européenne dans l'arrêt $V g T$, la Chambre des lords a privilégié la protection de l'opinion publique contre les influences indues d'une publicité politique partiale $^{71}$. Selon Lord Bingham, "the risk is that objects which are essentially political may come to be accepted by the public not because they are shown in public debate to be right but because, by dint of constant repetition, the public has been conditioned to accept them ${ }^{72}$.

Ce faisant, la Chambre des lords a fait siennes les conclusions du Tribunal fédéral suisse dans l'affaire $V g T$, considérant, en substance, que la Cour européenne n'avait pas apprécié cet argument à sa juste valeur ${ }^{73}$. La Chambre des lords a considéré, en outre, que l'interdiction était justifiée par un besoin social pressant dû à l'impact puissant de la publicité télévisuelle et radiophonique $^{74}$. Reste à savoir comment la Cour européenne statuera sur un éventuel recours, sachant que la Grande Chambre a confirmé, suite à l'inexécution de l'arrêt de 2001 par la Suisse, la violation de l'article 1o CEDH dans l'arrêt $V g T$ le 30 juin $2009^{75}$.

\section{Les effets}

30. Les effets juridiques traditionnellement rattachés à la liberté d'expression mettent à la charge des pouvoirs publics l'obligation de s'abstenir dans l'exercice de cette

66. 408 U.S. 665 (1972) Branzburg v. Hayes.

67. Cour EDH, Goodwin c. Royaume-Uni, 27 mars 1996, Rec., 1996-II.

68. ATF $132 \mathrm{I} 181 X$.

69. Cour EDH, Verein gegen Tierfabriken Schweiz c. Suisse, 28 juin 2001, Rec., 2001-VI; M. Hertig Randall et X.-B. Ruedin, «L'exécution des arrêts de la Cour européenne des Droits de l'homme à la lumière de l'arrêt Verein gegen Tierfabriken Schweiz (VGT) c. Suisse du 4 octobre 2007 ", PJA, 2008 , p. 651.

70. $R$ (on the application of Animal Defenders International) (Appellants) v. Secretary of State for Culture, Media and Sport (Respondent), [2008] UKHL 15.

71. Voir pour plus de détails sur les instances inférieures à la Chambre des lords, E. Barendt, «Political advertising on UK television», Medialex, 2007, p. 60 .

72. $R$ (on the application of Animal Defenders International) (Appellants) v. Secretary of State for Culture, Media and Sport (Respondent), [2008] UKHL 15, $\$ 28$.

73. Lord Bingham s'est exprimé ainsi : "I do not think the strength of this argument was deployed in VgT», $R$ (on the application of Animal Defenders International) (Appellants) v. Secretary of State for Culture, Media and Sport (Respondent), [2008] UKHL 15, \$22.

74. Là encore, l’argument avait déjà été soulevé, en vain, par le Tribunal fédéral : «Le Tribunal fédéral a expliqué à cet égard dans son arrêt du 20 août 1997 que la télévision avait un impact plus puissant sur le public en raison de son caractère général et immédiat. Cependant, de l'avis de la Cour, si les autorités nationales peuvent avoir des raisons valables de mettre en place cette différence de traitement, l'interdiction de la publicité à caractère politique qui ne s'applique qu'à certains médias et non à d'autres ne semble pas procéder d'un besoin particulièrement impérieux. » Cour EDH, Verein gegen Tierfabriken Schweiz c. Suisse, 30 juin 2009, $\$ 74$.

75. Cour EDH, Verein gegen Tierfabriken Schweiz c. Suisse, 30 juin 2009; voir M. Hertig Randall et X.-B. Ruedin, «Exécution des arrêts de la Cour européenne des Droits de l'homme», PJA, 2010, p. 116 sq. 
garantie comme le proclame par exemple l'article 11 de la Déclaration française de 1789 . Figure classique et incontestée du statut négatif caractéristique des libertés, cette conception ne suffit plus à épuiser, de nos jours, le champ opératoire de la liberté d'expression.

31. Conformément à la jurisprudence de la Cour européenne des Droits de l'homme, aux obligations négatives propres aux libertés, peuvent de nos jours s'ajouter des obligations positives, impliquant un devoir d'intervention à la charge des pouvoirs publics. L'obligation de respecter les libertés se double ainsi d'une obligation de protéger activement ces garanties, aux fins de leur conférer leur plein effet utile. La liberté d'expression n'échappe pas à cette vision novatrice.

La liberté d'expression constituant l'une des conditions préalables au bon fonctionnement de la démocratie, l'exercice réel et effectif de cette liberté ne dépend pas simplement du devoir de l'État de s'abstenir de toute ingérence. Il peut au contraire exiger également l'adoption de mesures positives. Dans le second arrêt $V g T$ rendu en 2009, la Cour européenne des Droits de l'homme a jugé que, compte tenu de l'importance de l'exécution effective des arrêts qu'elle rend, la Suisse avait l'obligation d'exécuter de bonne foi l'arrêt de 2001 en se conformant tant à ses conclusions qu'à son esprit. À cet égard, la réouverture de la procédure interne avait certes constitué une démarche importante aux fins de l'exécution de l'arrêt, mais elle ne saurait être considérée comme une fin en soi. En l'absence de motifs nouveaux pouvant justifier, dans le cadre de l'article 1o $\mathrm{CEDH}$, le maintien de l'interdiction de diffuser le spot télévisé dénonçant les conditions d'élevage de porcs en batterie, les autorités suisses avaient l'obligation d'autoriser la diffusion de ce spot, sans par ailleurs substituer leur jugement à celui de la société requérante quant à la persistance d'un intérêt du public pour le débat en question ${ }^{76}$.

32. La Cour de Strasbourg rappelle, au surplus, que l'article $10 \mathrm{CEDH}$ s'impose non seulement dans les relations entre employeur et employé soumises au droit public, mais peut également s'appliquer jusque dans les relations entre particuliers.

La Cour a en conséquence conclu à la violation de l'article 10 dans l'affaire Fuentes Bobo c. Espagne relative au licenciement d'un réalisateur d'émissions de télévision, à la suite des propos critiques que celui-ci avait tenus à l'égard de sa direction ${ }^{77}$.

\section{Les fonctions}

33. La liberté d'expression assume diverses fonctions. Il y a, bien entendu, la fonction de protection de l'individu par laquelle cette garantie est probablement la plus connue. La liberté d'expression est également dotée d'une importante fonction institutionnelle. Cette dernière joue le rôle fondateur de garantie de pluralisme, de tolérance et de multiplication des échanges d'idées au sein de la société civile. Tant il est vrai que l'expression détachée d'une perspective de communication paraît difficilement imaginable ${ }^{78}$. Le rôle sociétal de la liberté d'expression s'avère ainsi particulièrement marqué. Cette fonction sert à son tour de justification au régime démocratique.

Au Canada, la Cour suprême considérait, en 1938 déjà, la liberté en matière politique comme «le souffle de vie de la démocratie parlementaire ${ }^{79}$.

En France, le Conseil constitutionnel confère à la liberté d'expression le caractère de liberté fondamentale, une liberté « d'autant plus précieuse que son exercice est l'une des garanties essentielles du respect des autres droits et libertés et de la souveraineté nationale ${ }^{80}$. Pour les magistrats du Palais royal, le respect du pluralisme constitue l'une des conditions de la démocratie ${ }^{81}$.

Pour le Tribunal fédéral suisse, «la liberté d'expression n'est pas seulement, comme d'autres libertés expresses ou implicites du droit constitutionnel fédéral, une condition de l'exercice de la liberté individuelle et un élément indispensable à l'épanouissement de la personne humaine; elle est encore le fondement de tout État démocratique: permettant la libre formation de l'opinion, notamment de l'opinion politique, elle est indispensable au plein exercice de la démocratie. Elle mérite dès lors une place à part dans le catalogue des droits individuels garantis par la constitution et un traitement privilégié de la part des autorités ${ }^{82}$.

34. La Cour européenne des Droits de l'homme a, elle aussi, consacré de fort belles pages à la fonction institutionnelle impartie à la liberté d'expression dans les sociétés contemporaines.

La liberté d'expression constitue, selon les juges alsaciens, «l'un des fondements essentiels d'une société démocratique, l'une des conditions primordiales de son progrès; elle vaut non seulement pour les informations ou idées accueillies avec faveur ou considérées comme inoffensives ou indifférentes, mais aussi pour celles qui

76. Cour EDH, Verein gegen Tierfabriken Schweiz c. Suisse, 30 juin 2009.

77. Cour EDH, Fuentes Bobo c. Espagne, 29 février 2000 ; voir aussi Aguilera Jiménez et autres c. Espagne, 8 décembre 2009.

78. P. Wachsmann, «Liberté d'expression », p. 630.

79. Re Alberta Statutes, (1938) RCS 100.

80. Décision des 10 et 11 octobre 1984 relative à la loi visant à limiter la concentration et à assurer la transparence financière et le pluralisme des entreprises de presse.

81. Décision du 27 février 2007 concernant la loi relative à la modernisation de la diffusion audiovisuelle et à la télévision du futur.

82. ATF 96 I 586 Aleinick. Était en cause dans cette affaire la condamnation d'une personne qui avait distribué des tracts sur la voie publique, devant une entreprise, sans avoir sollicité d'autorisation au préalable. Après avoir mis en doute la question d'un usage accru du domaine public par une personne isolée, le Tribunal fédéral a admis le recours dirigé contre l'amende infligée en se fondant précisément sur le caractère privilégié de la liberté de la presse, fondement de tout État démocratique, lorsque celle-ci s'exerce sur le domaine public en vue de la distribution gratuite d'imprimés poursuivant un but idéal. 
heurtent, choquent ou inquiètent ; ainsi le veulent le pluralisme, la tolérance et l'esprit d'ouverture sans lesquels il n'est pas de société démocratique ${ }^{83}$. " La Cour constitutionnelle belge partage la même approche ${ }^{84}$.»

35. Garante de pluralisme, la liberté d'expression, au-delà des arbitrages entre majorité et minorité propres au système politique, assure une fonction fondamentale de capacité d'existence et d'expression critique en faveur des groupements les plus divers, peu ou insuffisamment représentés dans le processus d'adoption des lois et de conduite des affaires publiques. Elle a vocation à ménager, en d'autres termes, un puissant rempart en faveur de la protection de minorités.

À l'occasion d'un arrêt rendu en 1975, qui portait sur la diffusion d'un film présentant une nouvelle méthode d'interruption volontaire de grossesse dans un canton suisse à dominante catholique, le Tribunal fédéral a condamné l'interdiction qui avait frappé le film en question. Pour les juges fédéraux, particulièrement en verve ce jour-là, "en principe, dans une démocratie, chacun a le droit d'exposer ses vues sur un sujet d'intérêt public, même si elles déplaisent à certains. La majorité ne peut réduire la minorité au silence ${ }^{85}$.

36. L'arbitrage entre majorité et minorité n'est pas simple pour autant. Il fait appel à une approche d'évaluation et de pondération typique des modalités entourant l'admissibilité de restrictions au champ opératoire des libertés.

Le Tribunal fédéral suisse a, par exemple, souligné que la liberté d'expression ne doit pas revêtir une signification si étendue que le souci de lutte contre la discrimination raciale soit vidé de sa substance. Il doit, au contraire, être possible, dans une démocratie, de critiquer aussi le comportement de groupes humains déterminés. L'infraction n'est pas réalisée chaque fois qu'une personne tient un propos négatif à l'égard d'un groupe protégé par la norme pénale, pour autant toutefois que la critique reste globalement objective. Dans le débat politique, notamment dans le contexte d'élections, la liberté d'expression commande de ne pas admettre facilement l'existence d'un abaissement ou d'une discrimination répréhensibles au sens du droit pénal. Ainsi, les déclarations qui expriment certaines inégalités spécifiques et qui ne comportent pas, de façon explicite ou implicite, d'affirmation d'inégalité de droit à jouir des Droits de l'homme ne sont pas réputées rabaissantes ou discriminatoires. Tel est le cas lorsque l'affirmation semble xénophobe, de mauvais goût, amorale, choquante sur le plan moral ou encore inconvenante ou même non civilisée. Les messages concernant des questions politiques et des problèmes relevant de la vie publique revêtent une importance particulière dans ce contexte.
Les juges fédéraux avaient pour la circonstance à statuer sur des affiches qui montraient, par le biais d'un montage photographique, des musulmans accroupis en train de prier face contre terre devant le Palais fédéral, à Berne. Les affiches, présentées dans le cadre de la campagne pour les élections au Parlement fédéral de 2007, étaient accompagnées du slogan "Utilisez vos têtes! Votez UDC. Suisse, toujours libre!». Pour le Tribunal fédéral, les messages concernant des questions politiques et des problèmes relevant de la vie publique revêtent une importance particulière dans ce contexte. Dans une démocratie, il est en effet primordial de pouvoir défendre des points de vue qui déplaisent à une majorité et qui sont choquants pour de nombreuses personnes. La critique doit ainsi être admise dans une certaine mesure et parfois aussi sous une forme outrancière ${ }^{86}$.

37. La stimulation du débat, la capacité de penser, la recherche de la vérité et le renforcement de la cohésion politique et sociale en vue du bien commun constituent certaines des fonctions centrales de la liberté d'expression. Celle d'un marché des idées (marketplace of ideas) ouvert à la concurrence, un marché de libre compétition entre les idées et les opinions, où la seule force existante devrait être celle du meilleur argument, comme l'a souligné le juge américain Oliver Wendell Holmes dans l'opinion dissidente jointe à un arrêt rendu en 1919 par la Cour suprême ${ }^{87}$.

La consolidation de la démocratie à travers la participation de l'ensemble des citoyens en vue de favoriser l'émergence des jugements les plus bénéfiques pour la communauté permet d'expliquer pourquoi le discours politique bénéficie aux États-Unis d'une protection quasi absolue ${ }^{88}$.

38. Comme le relève le professeur Francis Delpérée, la liberté d'expression est la mère des libertés et, en ce sens, la mère de la démocratie. Si elle est bâillonnée, si elle est entravée à l'excès, il n'est pas d'exercice possible du débat et de la discussion qui est l'essence même de la vie d'une société démocratique ${ }^{89}$.

En Belgique, des restrictions ne peuvent être imposées au droit des citoyens d'exprimer leurs opinions, fûtce sur le ton polémique qui peut caractériser le débat public concernant des phénomènes de société, même lorsque ces opinions choquent, inquiètent ou heurtent l'État ou l'un ou l'autre groupe de la population. L'expression d'une opinion reste libre, même si elle est vive, critique ou polémique ${ }^{90}$.

39. Dans la même veine, la Cour européenne des Droits de l'homme accorde un droit à l'exagération et à la provocation en tant que composantes inhérentes au discours politique. Pour atténuer l'impact de cette

83. Cour EDH, Handyside c. Royaume-Uni, 7 décembre 1976, série A, nº 24, 449.

84. Arrêts $45 / 96$ et $157 / 2004$.

85. ATF 101 Ia 252 Ernst.

86. Arrêt du Tribunal fédéral 6B_664/2008 du 27 avril 2009, Procureur général du canton du Valais et ministère public du Bas-Valais.

87. 250 U.S. 616, 630 Abrams v. United States.

88. L. Pech, «Constitution et liberté d'expression... », p. 184.

89. F. Delpérée, «Constitution et liberté d'expression... », p. 145

90. Arrêt 157/2004. 
conception, la Cour a développé la théorie de la base factuelle, dont le fondement s'inscrit dans la distinction entre faits et jugements de valeur. Alors qu'une preuve peut être exigée pour les premiers, l'exactitude des seconds ne se prête pas à démonstration. En réalité, l'approche de la Cour est plus nuancée en ce qu'elle exige une base factuelle suffisante à l'appui du jugement de valeur, «faute de quoi elle serait excessive». Se pose dès lors la question du degré d'exigence qui peut être requis à l'auteur d'un jugement de valeur ${ }^{91}$.

\section{Les conditions d'exercice}

40. Pour certes protéger prioritairement l'individu, la liberté d'expression ne se décline pas exclusivement sur un ton isolé. Bien souvent, l'efficacité du message est liée aux conditions entourant sa diffusion. L'expression collective d'idées se présente ainsi comme un puissant vecteur qui sous-tend l'exercice de cette garantie. La liberté d'expression entretient ainsi inévitablement des rapports étroits avec les réunions et les manifestations sur le domaine public.

En France par exemple, le Conseil constitutionnel a rattaché à la liberté d'expression la liberté de manifester comme droit d'expression collective des idées et des opinions ${ }^{92}$.

41. La distinction entre réunion et manifestation est de nos jours dénuée de pertinence au regard de la liberté d'expression. En cas d'interdiction, les conditions de restriction posées par l'article 11 paragraphe $2 \mathrm{CEDH}$, plus particulièrement l'exigence de nécessité dans une société démocratique, doivent impérativement être respectées, au même titre qu'à l'article 10 paragraphe $2 \mathrm{CEDH}$.

Les lieux publics sont des endroits naturels et adéquats pour permettre la diffusion d'informations et d'opinions. La Cour suprême américaine l'a admis de longue date, en précisant toutefois que le respect de l'intérêt général pouvait justifier le respect de certaines conditions liées à l'horaire, au lieu et même à la forme des manifestations. Cette pratique est résumée sous la dénomination de "time, place and manner test ${ }^{93}$.

42. La réglementation des biens publics doit être neutre, sous l'angle du contenu des idées et des messages véhiculés. Aux États-Unis par exemple, les autorités ne sont, au nom du premier amendement, légitimées à faire prévaloir aucune espèce de faveur ou de défaveur en fonction des messages émis. La pesée des intérêts entre les positions qui s'affrontent doit en particulier prendre en compte la possibilité d'alternative en d'autres lieux. Dans ce domaine, le principe de la proportionnalité permet d'opérer la pesée des intérêts et, au besoin, de résoudre les cas de conflits de libertés susceptibles d'opposer la liberté d'expression à d'autres garanties.

La Cour suprême a, par exemple, jugé que la réglementation d'une petite ville du Wisconsin interdisant l'organisation de manifestations devant le domicile de médecins pratiquant l'avortement respectait la liberté d'expression, dès lors qu'elle laissait possible le déroulement de manifestations dans d'autres aires géographiques $^{94}$.

43. Les lieux visés par l'exercice de la liberté d'expression sont, à teneur de la jurisprudence de la Cour suprême américaine, les lieux traditionnellement ouverts au public comme les rues, les parcs ainsi que les alentours des bâtiments officiels. D’autres biens étatiques dépourvus de vocation de public forum ne sont pas exclus pour autant - comme les tribunaux, les abords d'établissements pénitentiaires ou les bases militaires. L'expression collective d'idées ou la diffusion de messages par voie de manifestations y sont toutefois soumises à des restrictions plus marquées et peuvent même y être totalement interdites ${ }^{95}$.

En Suisse, doctrine et jurisprudence admettent que la liberté de réunion peut être restreinte par l'application de la clause générale de police. Cette habilitation constitutionnelle implicite confère à l'autorité exécutive le droit, sans base normative expresse, de prendre les mesures indispensables pour rétablir l'ordre public s'il a été troublé, ou pour le préserver d'un danger sérieux qui le menace d'une façon directe et imminente ${ }^{96}$.

44. Au prix d'une évolution jurisprudentielle qui s'est étendue sur plusieurs années, le Tribunal fédéral suisse admet de nos jours que le particulier bénéficie d'un certain droit à l'usage accru du domaine public pour y exercer sa liberté d'expression. Le régime de l'autorisation a été jugé conforme à cette garantie, pour autant que l'autorité appelée à la délivrer ne s'en tienne pas aux idées et aux objectifs des organisateurs d'une manifestation. Là aussi, l'approche administrative doit être neutre du point de vue des messages véhiculés. L'installation de stands sur le domaine public implique un usage accru de celui-ci et peut dès lors être soumis à autorisation. Mais l'autorité doit prendre la liberté d'expression dans la pesée objective des intérêts à laquelle elle doit procéder lorsqu'elle statue sur l'octroi de l'autorisation ${ }^{97}$.

45. À partir de quel instant un usage commun du domaine public, libre, égal et gratuit pour tous, perd-il cette qualité pour devenir un usage accru soumis à autorisation? Cette question se résout en fonction des

91. La jurisprudence de la Cour est fluctuante sur ce point, comme en témoigne l'arrêt de la Grande Chambre dans la cause qui a opposé Lindon, Otchakovsky-Laurens et July à la France. Voir T. Hoffmann, «Diffamation et fiction», Medialex, 2008, p. 56. P. Wachsmann, «Vers un affaiblissement de la protection..." ", p. 491.

92. Décision du 18 janvier 1995 concernant la loi d'orientation et de programmation relative à la sécurité.

93. 461 U.S. 171 (1983) United States v. Grace; 408 U.S. 104 (1971) Grayned v. City of Rockford.

94. 487 U.S. 474 (1988) Frisby v. Schultz.

95. 385 U.S. 39 (1966) Adderley v. Florida; 424 U.S. 828 (1976) Greer v. Spock

96. ATF 103 Ia 310 Rassemblement jurassien et Unité jurassienne.

97. ATF 105 Ia 91 Plüss. 
standards propres au droit administratif. La liberté d'expression a toutefois son mot à dire, et son invocation ne manque pas d'influencer la conception contemporaine des biens de l'État.

En Suisse, il est admis que lorsque seules deux à trois personnes se rendent sur la voie publique pour y collecter des signatures à l'appui d'une initiative populaire tendant à la révision de la Constitution sans y installer de stand, l'usage du domaine public auquel elles se livrent ne cesse pas d'être commun. Les punir au motif qu'elles n'ont pas sollicité d'autorisation auprès de la commune concernée contrevient, partant, à la liberté d'expression ${ }^{98}$.

\section{Les restrictions}

46. Comme toute liberté, la liberté de s'exprimer n'est pas absolue. Les formules entourant sa capacité à être limitée sont innombrables, au gré des affaires déférées aux juges et de l'inspiration de ces derniers.

Dans un arrêt rendu en 1927, la Cour suprême américaine s'est, par exemple, exprimée dans les termes suivants: "the freedom of speech which is secured by the Constitution does not confer an absolute right to speak, without responsibility, whatever one may choose, or an unrestricted and unbridled license giving immunity for every possible use of language and preventing the punishment of those who abuse this freedom ${ }^{99}$."

47. Le régime des restrictions opposables à la liberté d'expression est lui-même évolutif, soulignant à la fois l'ampleur des modifications que peut subir la liberté au cours des âges et l'importance du contrôle judiciaire entourant sa mise en œuvre.

Dans un arrêt rendu le 16 mars 1971, la Cour constitutionnelle italienne a considéré que la norme pénale interdisant la discussion publique sur les sujets concernant la procréation ne pouvait être maintenue en vigueur sans violer la Constitution. L'évolution du débat relatif au problème de la limitation des naissances et le développement progressif de l'éducation sanitaire ne permettent plus de considérer comme une offense aux bonnes mours la discussion publique sur les pratiques anticonceptionnelles ${ }^{100}$. Dans un arrêt rendu en 1965, la Cour constitutionnelle avait pourtant rejeté les critiques dirigées contre cette disposition ${ }^{101}$.

48. Les restrictions aux libertés ne sont admissibles que si elles se fondent sur la loi. Cette condition comprend non seulement l'exigence d'une base légale en droit interne, mais vise également la qualité de la loi en cause. Ainsi, la loi doit être accessible au justiciable et prévisible dans ses effets.
Dans l'affaire Gsell c. Suisse, la Cour européenne des Droits de l'homme a estimé que la clause générale de police sur laquelle s'étaient fondées les autorités du canton des Grisons pour renvoyer un journaliste alors que celui-ci s'apprêtait à rédiger un article sur les manifestations à Davos dans le cadre du World Economic Forum et leurs effets sur la restauration et l'hôtellerie locales, n'était pas suffisamment précise. Selon les juges de Strasbourg, il appartenait aux autorités d'édicter une législation interne spécifique, susceptible de réglementer les manifestations qui se déroulaient, chaque année, dans le contexte du World Economic Forum ${ }^{102}$.

49. Le principe de la proportionnalité occupe une place centrale dans l'arbitrage, effectué par le juge, des motifs susceptibles de légitimer des restrictions à la liberté d'expression.

Dans l'arrêt Monnat c. Suisse, la Cour européenne des Droits de l'homme avait à se prononcer sur l'admission de plaintes dirigées contre une émission télévisée mettant en cause l'attitude de la Suisse durant la seconde guerre mondiale. Pour la Cour, compte tenu notamment de l'intérêt de la société démocratique à assurer la liberté d'expression, de la marge d'appréciation réduite s'agissant des informations d'intérêt général, du fait que la critique visait en l'espèce les agissements de hauts fonctionnaires gouvernementaux et d'hommes politiques ainsi que de la nature sérieuse du reportage litigieux et des recherches sur lesquelles ce dernier s'appuyait, l'admission de plaintes par les autorités suisses ne représentait pas un moyen raisonnablement proportionné à la poursuite du but légitime visé. Partant, l'article $10 \mathrm{CEDH}$ avait fait l'objet d'une violation ${ }^{103}$.

50. Une figure classique, mais néanmoins relativement complexe, des restrictions à la liberté d'expression met en cause les conflits de libertés. On entend par là l'hypothèse de l'invocation de la liberté d'expression par un justiciable, qui se heurte simultanément à la liberté d'un autre justiciable.

En Suisse, le Tribunal fédéral a par exemple précisé que les allégations attentatoires à l'honneur émanant d'un avocat au cours d'un procès pouvaient s'avérer justifiées par le devoir de plaider la cause et par le devoir professionnel, pour autant qu'elles soient pertinentes, qu'elles n'aillent pas au-delà de ce qui est nécessaire, qu'elles ne soient pas inutilement blessantes et ne soient pas propagées de mauvaise foi. En l'occurrence, les juges fédéraux ont considéré que l'allégation d'un avocat, selon laquelle les moyens utilisés par la partie adverse n'étaient "pas légaux», pouvait se justifier au cours d'une plaidoirie relative à l'attribution de la garde d'un enfant $^{104}$. 
51. Liberté d'expression et protection de la vie privée ont toujours baigné dans une certaine tension et entretenu des rapports conflictuels. Les juridictions nationales et régionales ont abordé ce type de conflit sous des angles très divers.

Aux États-Unis, la liberté d'expression garantie par le premier amendement occupe une place privilégiée. Le droit du public à l'information l'emporte ainsi généralement sur la protection de la vie privée ${ }^{105}$

De manière similaire, les tribunaux allemands accordent une protection très étendue à la liberté d'expression, parfois au détriment du droit au respect de la vie privée. Il suffit, pour justifier une intrusion dans la vie privée, que la victime de l'atteinte constitue une personnalité « absolue» de l'histoire contemporaine. La protection de ces personnes se limite aux seuls espaces privés, à moins que la personnalité ne se soit retirée dans un endroit isolé, à l'abri des médias.

La Cour européenne des Droits de l'homme a eu l'occasion de se prononcer sur l'approche des juridictions allemandes dans une affaire concernant la publication de clichés relatant des scènes privées de la vie quotidienne de la princesse Caroline Von Hannover ${ }^{106}$. De l'avis de la Cour, peu importe que la personnalité se trouve dans un lieu public ou confinée dans sa résidence privée, l'élément déterminant est celui de la contribution que les photos ou articles publiés apportent au débat d'intérêt général. En l'espèce, une telle contribution faisait défaut, la requérante ne remplissant pas de fonctions officielles et les photos litigieuses se rapportant exclusivement à des détails de sa vie privée ${ }^{107}$.

Cet arrêt a marqué un tournant dans la protection de la sphère privée, notamment au Royaume-Uni. L'arrêt Michael Douglas, Catherine Zeta-Jones, OK! contre Hello! rendu en 2005 en illustre l'impact ${ }^{108}$. Cette affaire, jugée en deux temps, concernait une requête en mesures provisionnelles visant à faire cesser la publication de photographies de mariage non autorisées par les époux Douglas et Zeta-Jones. La Cour d'appel de la Chambre des lords a, dans un premier temps, débouté les requérants, estimant en substance que la protection de la sphère privée devait céder le pas devant la liberté d'expression. Cing ans plus tard, la Cour d'appel est revenue sur sa décision, considérant que les juges saisis en 2000 n'avaient pas pu bénéficier de l'enseignement à tirer de l'arrêt Von Hannover ${ }^{109}$.

L'arrêt Otto-Preminger-Institut portait sur une plainte pénale déposée par le diocèse de l'Église catholique romaine d'Innsbruck pour dénigrement des doctrines religieuses contre la projection d'un film satirique, tournant en dérision les dogmes de la religion catholique, qui avait été ressenti comme blasphématoire. La plainte avait conduit à la saisie, puis à la confiscation du film ${ }^{110}$.

Ici, la Cour de Strasbourg retient, dans un premier temps, la protection des droits et libertés d'autrui comme motif légitime permettant de justifier la restriction à la liberté d'expression de l'association culturelle qui entendait projeter le film. Au sujet de la proportionnalité, elle relève certes que la question dont elle est saisie implique une mise en balance d'intérêts contradictoires tenant à l'exercice de deux libertés garanties par la Convention, les articles 9 et $10 \mathrm{CEDH}$. Soulignant l'atteinte injurieuse portée à la conception du public tyrolien au sujet de la religion catholique romaine, la Cour juge ensuite que la protection de «l'immense majorité des Tyroliens» et la nécessité de «protéger la paix religieuse dans cette région » constituent un motif nécessaire de restriction de la liberté d'expression de la requérante ${ }^{111}$.

52. La détermination précise du motif de restriction ne revêt pas qu'un caractère théorique ou académique. Elle conditionne de manière déterminante le regard ensuite porté par le juge, au regard de l'exigence de nécessité dans une société démocratique, sur les moyens avancés pour assurer sa réalisation.

Dans l'arrêt Otto-Preminger-Institut, la généralité des intérêts retenus au chapitre de la nécessité de la restriction à la liberté d'expression procède à notre sens de considérations insuffisamment individualisées pour relever de la protection des droits d'autrui au sens de l'article 10 paragraphe $2 \mathrm{CEDH}$. Toute respectable qu'elle est, la conception du public tyrolien en matière religieuse dans son ensemble relève en effet clairement d'un intérêt général - la protection de la conception morale propre à une religion - et non de l'appréciation ou de la perception propre à certaines personnes en particulier, confrontées à l'objet de la restriction.

L'arrêt marque ainsi une évidente contradiction sur le plan méthodologique entre le motif retenu pour justifier la restriction et l'examen de sa nécessité dans une société démocratique, qui affecte sa crédibilité. Ainsi considérée, l'affaire Otto-Preminger-Institut ne relevait pas d'un conflit de Droits de l'homme mais, plus classiquement, d'un simple cas de restriction à la liberté d'expression fondée sur l'intérêt public ${ }^{112}$.

53. Pilier à la fois de la liberté et de la démocratie, la liberté d'expression joue un rôle fondateur dans

105. Voir les exemples dans S. Bondallaz, La protection des personnes et de leurs données dans les télécommunications: analyse critique et plaidoyer pour un système en droit suisse, Zurich, Schultess Verlag, 2007.

106. Cour EDH, Von Hannover c. Allemagne, 24 juin 2004, Rec., 2004-VI.

107. Voir M. Bianchi Della Porta, «Information sur les personnalités, personnalisation de l’information: où sont les limites?», sic!, 2007, p. 507.

108. Voir E. Barendt, «A right to privacy at last in English law? ", Medialex, 2006, p. 72.

109. "The Court of Appeal did not have the benefit of the reasoning in the House of Lords in Campbell v. MGN or, even more significantly for present purposes, the reasoning of the ECtHR in von Hannover v. Germany", Douglas v. Hello! Ltd v. OK! Catherine Zeta-Jones and OK! v. Hello [2005] EMLR 28.

110. Cour EDH, Otto-Preminger-Institut c. Autriche, 20 septembre 1994, série A, nº 295-A.

111. $\$ 56$ de l'arrêt.

112. Voir également le commentaire de P. Wachsmann, «La religion contre la liberté d'expression: sur un arrêt regrettable de la Cour européenne des Droits de l'homme. L'arrêt Otto-Preminger-Institut du 20 septembre 1994", Revue universelle des Droits de l'homme, vol. 6, nº 12, 1994, p. 441. 
l'agencement de l'État contemporain. Peut-être plus que toute autre, cette garantie traduit la relation triangulaire unissant l'individu, la société civile et les pouvoirs publics, traduisant d'une manière particulièrement éloquente la particularité propre aux libertés de ne pouvoir vivre et s'épanouir qu'en société, dans les rapports avec autrui. Les valeurs d'affranchissement, d'autonomie et de communication qu'elle véhicule restent d'actualité avec une constance étonnante.
54. Sa mise en œuvre par le juge a également rendu possible une évolution remarquable de la théorie des libertés et, plus largement, des droits fondamentaux dans leur ensemble. Preuve supplémentaire - si besoin est - que la justice constitutionnelle, à laquelle vient utilement s'ajouter la justice conventionnelle, assume, elle aussi, un rôle de premier plan dans le respect, la protection et la mise en œuvre des Droits de l'homme. 
